\section{Pseudo-Outbreak Associated With an Antibiotic Management Program}

Calfee et al. from the University of Virginia Health System, Charlottesville, Virginia, reported an abrupt and persistent $30 \%$ increase in the rate of nosocomial infections detected at a university teaching hospital after a prolonged period with a relatively constant nosocomial infection rate. Demographic data, risk factors for nosocomial infection, features of reported cases of nosocomial infection, and policy and procedure changes were evaluated for the periods of January 1, 1997, to April 30, 1998 (endemic period), and May 1 to December 31, 1998 (epidemic period). An extensive outbreak investigation revealed no evidence of a true outbreak of nosocomial infection. The apparent outbreak involved all four major body sites, began during the same month that an antibiotic management program was started, involved the same adult medical and surgical units where antibiotics were being controlled, and occurred months before any significant change in antibiotic use. The nosocomial infection surveillance nurses reported a greater proportion of nosocomial infection during the epidemic period, based on a treating physician's diagnosis rather than on specific clinical criteria. In an attempt to justify existing antibiotic prescribing practices after the implementation of an antibiotic management program, clinicians altered the threshold at which they documented the presence of nosocomial infection. This change in documentation produced a large pseudo-outbreak of nosocomial infection.

FROM: Calfee DP, Brooks J, Zirk NM, Giannetta ET, Scheld WM, Farr BM. A pseudo-outbreak of nosocomial infections associated with the introduction of an antibiotic management programme. J Hosp Infect 2003;55:26-32.

\section{Methicillin-resistant Staphylococcus aureus in Children With Cystic Fibrosis: An Eradication Protocol}

Solis and colleagues from Royal Liverpool Children's Hospital, National Health Service Trust,
Liverpool, United Kingdom, conducted a retrospective 12-year study from May 1988 to July 2000 in children with cystic fibrosis (CF) to evaluate (1) the magnitude of methicillin-resistant Staphylococcus aureus (MRSA) in these children; (2) the clinical impact of MRSA on CF; and (3) the efficacy of a MRSA protocol aimed at the eradication of the carrier state. The study maneuver was composed of (1) surveillance cultures of the throat or rectum to detect the MRSA carrier state; (2) lifelong cephradine rather than flucloxacillin to lift selection pressure; (3) topical application of oral and nebulized vancomycin for 5 days to clear the carriage of MRSA; and (4) a strict anti-staphylococcal hygiene program, including a hand washing and device policy. Fifteen children with CF (11 boys; median age, 117 months) positive for MRSA were enrolled. The current prevalence of MRSA among children with $\mathrm{CF}$ in the hospital was $6.5 \%$.

Of the 15 children identified, only 12 (18 episodes of MRSA colonization) were treated according to the protocol. The median age at MRSA acquisition was 73 months (interquartile range, 43 to 134 months). In $7(55 \%)$ of the patients, MRSA was eradicated. For a total of 18 MRSA episodes, the protocol was successful in 10 episodes. The mean period of MRSA-free status was 12 months (range, 6 to 36 months). Pulmonary function [measured by FEV(1)] was not affected ( $68 \%$ predicted before treatment, and $68 \%$ predicted after treatment). All children were oropharyngeal carriers of both MRSA and ceftazidime-resistant Pseudomonas aeruginosa.

The authors concluded that an effort to limit MRSA in $\mathrm{CF}$ clinics is needed because (1) MRSA carriage in any individual is an abnormal condition; (2) limitation of systemic vancomycin use is desirable; (3) MRSA carriage may be a contraindication for lung transplantation; and (4) epidemiologically, a CF unit with a substantial MRSA problem functions as a source of dissemination for other patients.

FROM: Solis A, Brown D, Hughes J, Van Saene HK, Heaf DP. Methicillin-resistant Staphylococcus aureus in children with cystic fibrosis: an eradication protocol. Pediatr Pulmonol 2003;36:189-195. 